\title{
Primary non-Hodgkin's lymphoma masquerading as cervical cancer
}

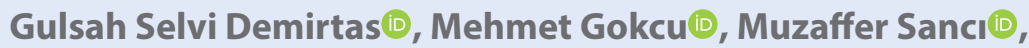 \\ Sumeyye Ekmekci@, Halil Ibrahim Yıldız@ \\ Tepecık Educatıon and Research Hospıtal, Izmır, Turkey
}

\begin{abstract}
Genital tract lymphomas are rare entities that can be diagnosed at advanced stages. The uterine cervix is not generally infiltrated by lymphoma. Nevertheless it can be seen as a consequence of either a systemic disease or primary disease. The infrequency of primary cervical lymphoma makes the diagnosis challenging.
\end{abstract}

Key words: uterin cerviks; Non Hodgin's Lymphoma; CIN 3

Ginekologia Polska 2020; 91, 9:571

Herein we report a case of 46-year-old woman, referred to our clinic because of vaginal bleeding. Physical examination revealed a cervical mass of approximately $10 \mathrm{~cm}$ in diameter that filled the vagina entirely. There was bilateral parametrial involvement, and this examination resembled cervical cancer. Colposcopy-directed punch biopsies resulted in a diagnosis of cervical intraepithelial neoplasia (CIN) 3. Subsequently, a cone biopsy was performed and histopathological examination revealed the diagnosis of diffuse B cell non-Hodgkin's lymphoma along with CIN 3. Tumor cells showed diffuse CD20, PAX5, CD79a positivity (Fig. 1, 2). Positron Emission Tomography (PET CT) showed a bulky cervical mass and with probable involvement of the parametrium. There was no evidence of extension to other sites.

The surgical margins of the cone biopsy were free of tumors. The patient was referred to the Medical Oncology Clinic for further evaluation. Bone marrow examination was normal. Depending upon the findings, a diagnosis of the primary cervical Non-Hodgkin's Lymphoma with diffuse large B cell type was made. The patient received three cycles of CHOP (Cyclophosphamide, adriamycin, vincristine, and prednisone ) chemotherapy. After three cycles of chemotherapy, the tumor completely disappeared. An additional three cycles of chemotherapy were administered. The patient was referred to the Radiation Oncology Department and treated with external beam radiotherapy (46 Gy). Radiographic and clinical findings of the disease resolved completely. For CIN 3 surveillance, the patient was observed with colposcopy and cytology in 6-months intervals.

The prognosis and treatment of primary cervical lymphoma is entirely different from the squamous cell carcinoma of the cervix. Although being rare, this diagnosis should be kept in mind in the differential diagnosis of cervical masses. The coexistence of CIN 3 and non-Hodgkin's lymphoma could be purely coincidental and an accompanying, cervical lymphoma can be overlooked, if biopsies were not adequately taken.

Based on the described clinical vignettes, cervical punch biopsy, especially cone biopsy and detailed immunohistochemical studies, are essential for cervical lymphoma diagnoses. In the treatment of cervical non-Hodgkin's lymphoma, combination radiotherapy with chemotherapy has a good response rate.

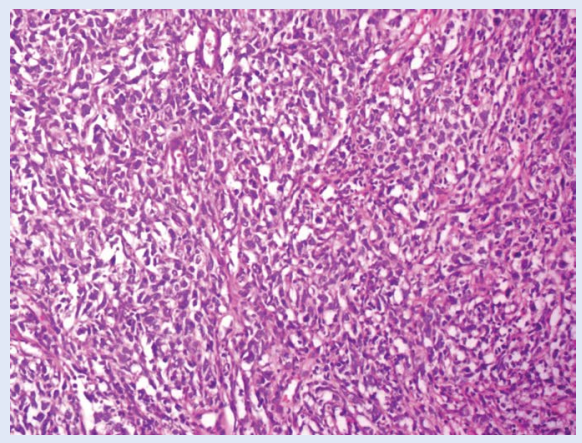

Figure 1. Diffuse large B-cell lymphoma infiltration in the cervix $(\mathrm{H} \& \mathrm{E}, \mathrm{x} 400)$

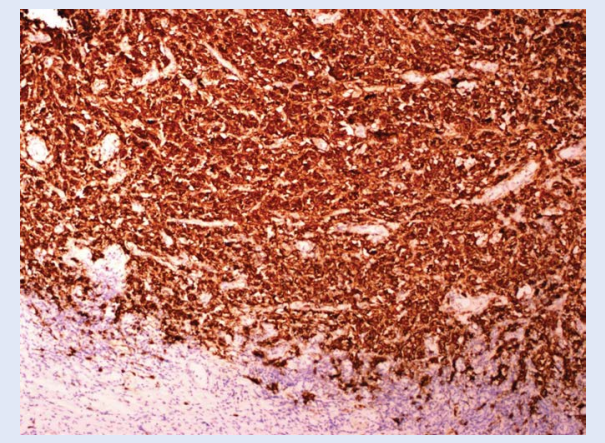

Figure 2. Immunohistochemically, tumor cells showed diffuse CD20 staining (DAP, x200)

Corresponding author:

Gulsah Selvi Demirtas

Tepecık Education And Research Hospıtal, Tepecık Educatıon And Research Hospıtal, 35500 Izmır, Turkey

e-mail:drg.selvi@gmail.com 